\title{
SOFTWARE ExergAn - ANÁLISE PRELIMINAR DE VIABILIDADE TERMODINÂMICA E ECONÔMICA DE CULTURAS AGRÍCOLAS GERADORAS DE BIOCOMBUSTÍVEIS
}

\author{
João Pedro Leal Maran'1, Cid Marcos Gonçalves Andrade², José Eduardo Olivo³ \\ ${ }^{1}$ Acadêmico do curso de Engenharia Química, Universidade Estadual de Maringá - UEM. Bolsista PIBITI/CNPQ-FA. \\ joaoplmaraneq@gmail.com \\ ²Coorientador, Doutor, Departamento de Engenharia Química, Universidade Estadual de Maringá - UEM. cmgandrade@uem.br \\ 3Orientador, Doutor, Departamento de Engenharia Química, Universidade Estadual de Maringá - UEM. jeolivo@uem.br
}

\section{RESUMO}

Como alternativa aos combustíveis fósseis, fontes energéticas renováveis têm sido uma opção cada vez mais utilizadas e pesquisadas. Além de suprir a necessidade energética das máquinas e equipamentos, elas diminuem o impacto ambiental gerado pela utilização de tais recursos, uma vez que fazem uso das espécies químicas já instauradas nos ciclos biogeoquímicos do planeta. No entanto, a escolha feita pelos produtores sobre quais culturas biomássicas plantar, sabendo que serão consumidas no mercado de combustíveis renováveis, e a escolha feita pelas indústrias bioenergéticas sobre quais delas utilizar em suas plantas nem sempre são as mais adequadas. Dessa forma, desenvolveu-se um software que efetua uma análise exergoeconômica sobre diferentes tipos de culturas agrícolas geradoras de biocombustíveis, levando em conta conceitos termodinâmicos e econômicos. A princípio, o software receberá informações sobre a plantação da cultura escolhida, e será necessário fazer também a escolha do tipo de processo que ser quer analisar. Em sequência, o programa fornecerá dois parâmetros, a saber, eficiência exergética e custo exergoeconômico, que fornecerão informações relevantes para comparar diferentes biocombustíveis provenientes de biomassas distintas, auxiliando as escolhas tomadas pelos produtores e pelas indústrias. Devido à grande variedade de culturas biomássicas e de processamentos industriais associados a suas transformações, escolheu-se limitar o software às produções de bioetanol de primeira geração e à produção de biodiesel a partir da soja, por serem os biocombustíveis mais conhecidos e representativos no Brasil.

PALAVRAS-CHAVE: Análise de viabilidades; Exergia; Exergoeconomia;

\section{INTRODUÇÃO}

A necessidade energética vem crescendo na sociedade desde seus primórdios. Após a revolução industrial, com o advento das máquinas e dos motores, os seres humanos reduziram o uso de tecnologias de produção manuais e pecuárias. Desde então, cada vez mais tecnologias vêm sendo desenvolvidas e, como consequência, os requisitos energéticos vêm aumentando também. Para que as necessidades energéticas sejam supridas, muitas vezes recursos naturais não renováveis são utilizados como matéria prima. O caráter finito dos recursos naturais e os impactos ambientais provocados pela exploração e consumo de petróleo, gás natural e carvão mineral deixa evidente que essas fontes deverão ser gradativamente abandonadas e que é indispensável investir seriamente no desenvolvimento de fontes de energia que sejam renováveis e limpas (Carvalho, 2014).

Um ramo da tecnologia da sociedade contemporânea que tem destaque pelo seu alto consumo de fontes não renováveis é o automobilismo. Nesse ramo a grande maioria dos veículos automóveis utilizam combustíveis derivados do petróleo, que é um óleo natural constituído em sua maior parte por hidrocarbonetos, fruto de um demorado processamento de matéria orgânica realizado, ao longo de milhares de anos, pela natureza. Sua fonte não é infinita, e seu uso terá que ser substituído eventualmente por outras fontes de energia. 
Uma alternativa é o uso de biocombustíveis, que são definidos como qualquer combustível de origem biológica com exceção da fóssil (Arredondo, 2009).

Muitos biocombustíveis são produzidos a partir de culturas biomássicas renováveis, tais como soja, cana-de-açúcar, milho, entre outras. Cada uma dessas biomassas servirá como matéria prima para um tipo específico de biocombustível. Alguns, como o biodiesel, podem ser obtidos também por gordura animal (Azevedo, 2016). Essa energia tem origem nas ligações químicas das moléculas, acumulada por seres vivos em seus ciclos biológicos. Assim, diferentes culturas serão transformadas em diferentes biocombustíveis, com distintas quantidades de energia disponíveis em suas ligações químicas.

A energia se manifesta de várias maneiras, cada uma delas com suas próprias características e com sua qualidade. A qualidade da energia é avaliada em termos de sua capacidade de causar mudança. Essa qualidade, para formas desordenadas de energia, caracterizadas pela entropia, é variável, enquanto que para formas ordenadas de energia, que não são caracterizadas pela entropia, é invariante. Dessa forma, as primeiras podem ser totalmente convertidas em outras formas de energia (Kotas, 1995).

Para considerar a qualidade variável de formas desordenada de energia, um parâmetro universal de qualidade é necessário. O padrão mais natural e conveniente é o máximo de trabalho que pode ser obtido de uma certa forma de energia dados os parâmetros do ambiente como estado de referência. Esse padrão é a chamada exergia (Kotas, 1995). Portanto, a exergia é apenas a parcela da energia total que realmente pode ser convertida em trabalho útil.

À perda de parte da exergia total associam-se as irreversibilidades do sistema, presentes em quase toda, senão toda, transformação físico-química na natureza, sendo a parcela de exergia restante no produto final realmente utilizável. Pela análise exergética avalia-se a eficiência da transformação dos reagentes biomássicos em energia útil, sendo essa ferramenta importante pois ela possibilita avaliar quantitativamente as causas que resultam em aumentos de exergia devido às imperfeições termodinâmicas dos processos térmicos e químicos (Szargut, 1989). Com essa eficiência, comparam-se as viabilidades termodinâmicas do processamento de diferentes culturas biomássicas. Assim, uma análise exergética da produção de um biocombustível consiste em quantificar o quanto de exergia alimentada a esse processo pode realmente ser convertida em exergia nos produtos, sendo ele principais ou secundários.

Conjuntamente à análise exergética, agregam-se dados econômicos do processo, implementando conceitos da exergoeconomia, uma das quatro principais metodologias de análise termoeconômica (Cerqueira, 1999). O intuito é obter uma informação valiosa sobre a produção do biocombustível: o custo exergoeconômico. Com ele, tem-se um dado que permite comparar o preço por unidade de energia de diferentes biocombustíveis provindos de variáveis plantas industriais, a fim de identificar qual é mais viável economicamente e termodinamicamente.

Com o intuito de dispor uma ferramenta que permita decidir qual o plantio mais eficiente ou qual a melhor rota industrial para a produção energética, a partir de um embasamento termodinâmico, desenvolveu-se um software que permitirá informar a eficiência exergética e o custo exergoeconômico da cultura biomássica tomada, a partir de dados de área de plantação, produtividade anual, custo total anual e de um parâmetro que relaciona as diferentes exergias da planta, para aqueles que possuem tal informação. 
Para a análise preliminar de viabilidade termodinâmica e econômica, que será realizada pelo algoritmo do software, algumas suposições foram tomadas:

- O reator químico de cada produção de biocombustível será o alvo fundamental da análise, de forma que os balanços mássicos e exergéticos serão realizados sobre ele, desconsiderando-se análises mais complexas sobre os processos de preparação da matéria prima, separação, purificação e afins.

- A alimentação do reator será considerada pura em reagentes. Sendo assim, todo material que entra no reator é reagente. A presença dos catalisadores também é desconsiderada nos cálculos de balanços.

- Os balanços exergéticos são tomados apenas sobre a exergia química das espécies envolvidas na reação, nas condições padrão de temperatura e pressão (Temperatura de $298,15 \mathrm{~K}$ e Pressão do reator igual a $1 \mathrm{~atm}$ ).

- Caso se queira contabilizar a influência de outros tipos de exergia que influenciem na exergia das correntes de entrada, sejam elas provenientes das utilidades ou das operações unitárias da linha de produção, define-se neste trabalho um parâmetro J, que relaciona de forma simplificada a exergia química alimentada no reator com as demais formas de exergia alimentadas no processo. Esse parâmetro será obtido a partir de um estudo exergético prévio sobre a planta industrial a ser analisada, que fornecerá um valor representativo do parâmetro J. Para as exergias dos produtos, será admitida apenas a saída de exergia química.

- A exergia gasta na plantação, assim como na colheita e no transporte de matérias primas ou de produtos, foi desconsiderada.

- O custo do processamento da matéria-prima até seu produto final não é discriminado de acordo com as especificações dos processos de transformação das biomassas, sendo utilizado diretamente o custo anual da produção do biocombustível como parâmetro de entrada.

- O alvo do programa é apenas o biocombustível produzido, sendo ele a única exergia dos produtos que é contabilizada no cálculo dos parâmetros de eficiência exergética e custo exergoeconômico. Portanto, é desconsiderada a utilidade e influência dos demais produtos, para que apenas a contribuição exergética do biocombustível produzido seja analisada. Assim, apesar da definição da eficiência exergética ser baseada na somatória de todas as exergias dos produtos sobre a somatória das exergias de entrada do processo, toma-se a soma das exergias dos produtos como sendo apenas a exergia do biocombustível produzido.

- O cálculo de custo exergoeconômico contabiliza toda a exergia útil do biocombustível, podendo ter seu valor alterado numa condição real em que se tenha uma eficiência do processo de liberação da exergia que se encontra nas moléculas do biocombustível. Um exemplo é um motor de combustão, no qual parte da energia útil é transformada em calor e em outras formas de energia. EXERGOECONÔMICO 
As exergias químicas específicas das moléculas, envolvidas nas reações químicas de produção do bioetanol e do biodiesel, são necessárias para o balanço exergético. Alguns dados de exergia química específica são obtidos de literaturas, podendo ser utilizados após uma simples conversão de unidades ou um equacionamento simples. O biodiesel e o óleo de soja tiveram suas exergias químicas específicas calculadas, uma vez que não foram encontrados esses dados em tabelas informativas. Para obter a exergia química do biodiesel, utiliza-se a seguinte correlação (Szargut, 1988):

$$
\varphi=\frac{b^{c h}}{P C I}(1)
$$

Sendo PCI o poder calorífico inferior, $b^{c h}$ a exergia química específica e $\varphi$ uma correlação específica para algumas substâncias biomássicas, como biodiesel e glicerina:[4]

$$
\varphi=1,0374+0,0159 \frac{h^{\prime}}{c^{\prime}}+0,0567 \frac{o \prime}{c^{\prime}}(2)
$$

A equação (1) também é utilizada para o cálculo da disponibilidade exergética da soja, tendo a seguinte correlação para $\varphi$ (Gomes, 2017):

$$
\varphi=\frac{1,0412+0,212\left(\frac{H}{C}\right)-0,249\left(\frac{O}{C}\right)\left[1+0,7884\left(\frac{H}{C}\right)\right]+0,045\left(\frac{N}{C}\right)}{\left[1-0,3035\left(\frac{O}{C}\right)\right]}
$$

Com $H, C, O$ e $N$ sendo as frações mássicas de hidrogênio, carbono, oxigênio e nitrogênio respectivamente. Assume-se que o balanço de massa se dá sem acúmulo, de forma que toda a massa que entra no reator sai dele.

O balanço de exergia, de forma simplificada, pode ser representado como:

$$
\sum\left(E_{x_{\text {entradas }}}\right)-\sum\left(E_{x_{\text {saídas }}}\right)=I(4)
$$

Com $E_{x_{\text {entradas }}}, E_{x_{\text {saídas }}}$ e $I$ sendo respectivamente as correntes exergéticas que entram no sistema, que saem do sistema, e as irreversibilidades exergéticas das operações unitárias/transformações que ocorrem ao longo da linha de produção industrial. $E_{x}$ pode ser expandida em função das componentes exergéticas químicas, físicas, cinéticas e potenciais (Kotas, 1995). Logo:

$$
E_{x}=\dot{m}\left(e^{c h}+e^{p h}+e^{k i n}+e^{p o t}\right)(5)
$$

A mais relevante para este trabalho é a química, tendo em vista que ela tem uma maior contribuição de forma geral. Além dela, a exergia física pode ser relevante, a depender da planta industrial; as exergias cinética e potencial costumam ser desprezadas em sistemas industriais. A eficiência exergética $(\varepsilon)$ é calculada como (Mari, 2018):

$$
\varepsilon=\frac{\sum\left(E_{x_{\text {produtos }}}\right)}{\sum\left(E_{x_{\text {entradas }}}\right)}(6)
$$

onde $E_{x_{\text {produtos }}}$ são as correntes exergéticas dos produtos, e $E_{x_{\text {entradas }}}$ são as correntes de exergia que entram no sistema. Define-se um parâmetro J para expressar, de forma mais simples, as exergias que entram no sistema:

$$
J=\left(\frac{e^{p h}+e^{k i n}+e^{p o t}}{e^{c h}}\right)_{e n t r a d a}
$$

Com esse novo parâmetro, pode-se analisar de forma mais simplificada a influência das exergias não químicas que entram no sistema. Desconsiderando as contribuições 
exergéticas física, cinética e potencial dos produtos, a eficiência exergética pode ser reescrita como:

$$
\varepsilon=\frac{\sum \dot{m}\left(e^{c h}\right)_{\text {produtos }}}{\sum \dot{m}\left(e^{c h}\right)_{\text {entrada }}(1+J)}(8)
$$

Para o trabalho, busca-se focar na produção do produto principal. Normalmente, seriam tomados os produtos como sendo todos os formados na reação química. No entanto, ao considerar o produto apenas o biocombustível, tem-se uma análise exergoeconômica específica da produção desse biocombustível. Assim, a eficiência exergética específica evidenciará a quantidade de exergia da cultura biomássica que foi transferida para o biocombustível, sendo essa eficiência exergética específica da produção do biodiesel apenas parte da eficiência exergética da reação química do reator. Representando matematicamente:

$$
\varepsilon=\sum \varepsilon_{\text {produto } i}=\sum \frac{\dot{m}\left(e^{c h}\right)_{\text {produtoi }}}{\sum \dot{m}\left(e^{c h}\right)_{\text {entrada }}(1+J)}(9)
$$
produto.

Com $\varepsilon_{\text {produto } i}$ sendo a eficiência exergética específica para a produção de um

Por fim, o custo exergoeconômico evidenciará o custo por unidade de energia disponível no biocombustível gerado. Ele será o parâmetro comparativo da análise exergoeconômica, calculado com a seguinte equação:

$$
C_{x c}=\frac{C_{\text {total }}}{\varepsilon \cdot E_{x_{\text {produtos }}}}(10)
$$

\subsubsection{Produção de Bioetanol de Primeira Geração}

O bioetanol de primeira geração é produzido a partir dos açúcares presentes na composição da matéria prima. Para a cana-de-açúcar, consideram-se $14 \%$ de massa de sacarose e $0,9 \%$ de glicose (Lima, 2001). A sacarose passa por uma hidrólise prévia à fermentação, sendo convertida em glicose e frutose, para finalmente ser alimentada no reator químico. Assume-se conversão igual a 90\% (Oliveira, 2013). A fermentação alcóolica, catalisada por enzimas das leveduras, segue a reação descrita abaixo:

$$
\text { Glicose } \rightarrow 2 \text { Etanol }+2 \text { Dióxido de carbono }
$$

Os balanços de massa e de exergia utilizarão dados de massas molares e exergias químicas específicas desses componentes. Para a produção de bioetanol, os dados são descritos na tabela a seguir:

Tabela 1: Massas molares e exergias químicas específicas das espécies químicas da produção de bioetanol, nas condições padrão

\begin{tabular}{c|cc} 
ESPÉCIES QUÍMICAS & $\begin{array}{c}\text { MASSA } \\
\text { MOLAR(kg/kmol) }\end{array}$ & $\begin{array}{c}\text { EXERGIAS QUÍMICAS } \\
\text { ESPECÍFICAS(kJ/kg) }\end{array}$ \\
\hline CANA-DE-AÇÚCAR & - & 5762 \\
GLICOSE & 180,156 & - \\
SACAROSE & 342,3 & - \\
ETANOL & 46,07 & 27154 \\
DIÓXIDO DE CARBONO & 44,01 & 451
\end{tabular}




\subsubsection{Produção de Biodiesel de Soja por Transesterificação}

O processo de transesterificação requer triglicerídeos e álcoois como reagentes. A cultura biomássica da soja apresenta 18-20\% de óleo de soja, sendo ele composto por 2,3$11 \%$ de ácido palmítico, 2,4-6\% de ácido esteárico, 23,5-31\% de ácido oleico, 49-51,5\% de ácido linoleico e 2-10,5\% de linolênico (Brandão, 2007). A transesterificação assumida é a de catálise alcalina, por ser a mais comum. O álcool admitido como reagente será o metanol ou o etanol. A princípio, a utilização do metanol apresenta uma maior simplicidade no processo; porém, é sintetizado a partir de fontes não renováveis e o país não tem autossuficiência em sua produção. Por outro lado, o Brasil é o maior produtor de etanol, sendo este provindo de fontes renováveis, o que torna sua escolha interessante apesar das dificuldades de processamento (Medeiros, 2014). Para ambos os casos, a reação de transesterificação do óleo de soja é:

$$
\text { Triglicerídeo }+3 \text { álcoois } \rightleftharpoons 3 \text { biodiesel }+ \text { Glicerol }
$$

No programa, será considerado apenas um dos tipos de álcool como reagente por análise. A razão molar de álcool/óleo é comumente de 6:1, sendo ela a padrão do software. Assume-se ainda uma conversão de $95 \%$ tanto para a via de álcool metílico quanto para a via de álcool etílico.

Dados das composições elementares da soja, do biodiesel e da glicerina são requeridos para o cálculo da exergia química específica. Estão representados na tabela abaixo:

Tabela 2: Composição elementar da soja, biodiesel e glicerina

\begin{tabular}{ccccc} 
& $\begin{array}{c}\text { CARBONO } \\
(\%)\end{array}$ & $\begin{array}{c}\text { HIDROGÊNIO } \\
(\%)\end{array}$ & $\begin{array}{c}\text { OXIGÊNIO } \\
(\%)\end{array}$ & $\begin{array}{c}\text { NITROGÊNIO } \\
(\%)\end{array}$ \\
\hline SOJA & 39,27 & 5,76 & 52,95 & 0,7 \\
BIODIESEL & 74,70 & 12,10 & 13,10 & - \\
GLICERINA & 39,13 & 8,75 & 52,12 & -
\end{tabular}

O poder calorífico inferior da soja, matéria-prima biomássica, é de $19400 \mathrm{~kJ} / \mathrm{kg}$, sendo estimada como 6\% abaixo do valor do poder calorífico superior (Mourad, 2011). Os dados que serão requisitados pelo software de exergias químicas e as massas molares foram calculados expostos na tabela 3.

Tabela 3: Massas molares e exergias químicas específicas das espécies químicas da produção de biodiesel de soja, nas condições padrão

\section{ESPÉCIES QUÍMICAS

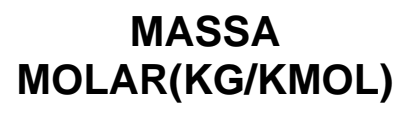

GRÃO DE SOJA
OLLEO DE SOJA
METANOL
ETANOL
BIODIESEL
GLICERINA

873,3

32,07

46,07

$295,6^{*}$

92,09 $\begin{array}{cc}\text { PODER } & \text { EXERGIAS } \\ \text { CALORÍFICO } & \text { QUÍMICAS } \\ \text { INFERIOR(KJ/KG) } & \text { ESPECIIFICAS(KJ/KG) }\end{array}$ 19400 22939

\section{9}

27154

37200 $39019^{\star *}$

*A massa molar do biodiesel de soja foi aproximada a partir da divisão das correntes mássicas e molares da tabela 1, encontrada em (Mari, 2018). ${ }^{* *}$ É suposto que as exergias específicas dos biodieseis produzidos via metílica e etílica são iguais. 
O SCILAB foi utilizado como base para o programa. O código de programação foi separado em três partes: a primeira com os cálculos das propriedades termodinâmicas e econômicas, a segunda com a interface gráfica, e a terceira com as funções que interligam a interface gráfica e valores de entrada do usuário com o cálculo termodinâmico. Quatro dados de entrada são fornecidos pelo usuário (Área total de cultivo, produtividade anual, custo total anual e parâmetro J), que juntamente com outros dados levantados para os processos de produção disponibilizados no programa, fornecem as duas variáveis de saída que permitem a comparação exergoeconômica entre diferentes processos de produção de biocombustíveis, no caso a eficiência exergética do biocombustível e seu custo exergoeconômico. Além disso, foi elaborado um arquivo de texto que traz algumas informações e responde algumas possíveis dúvidas do usuário, contendo também um tutorial básico de como utilizar o software.

A interface gráfica foi feita de forma simples e funcional, com intuito de que o cálculo preliminar possa ser obtido de forma rápida e direta. Ela possui uma lista de opções de processo, quatro espaços para valores de entrada e três botões (calcular, resetar e ajuda). A partir da escolha do processo e das entradas dos dados nos espaços respectivos, podese calcular o custo exergoeconômico e a eficiência exergética pressionando o botão calcular. O botão resetar zera os valores dos espaços preenchidos, para agilizar uma nova análise. Por fim, o botão ajuda abre uma caixa de mensagem que informa ao usuário que as dúvidas gerais são respondidas no arquivo ExergAn - readme, complementar ao software. A figura a seguir mostra a janela do programa:

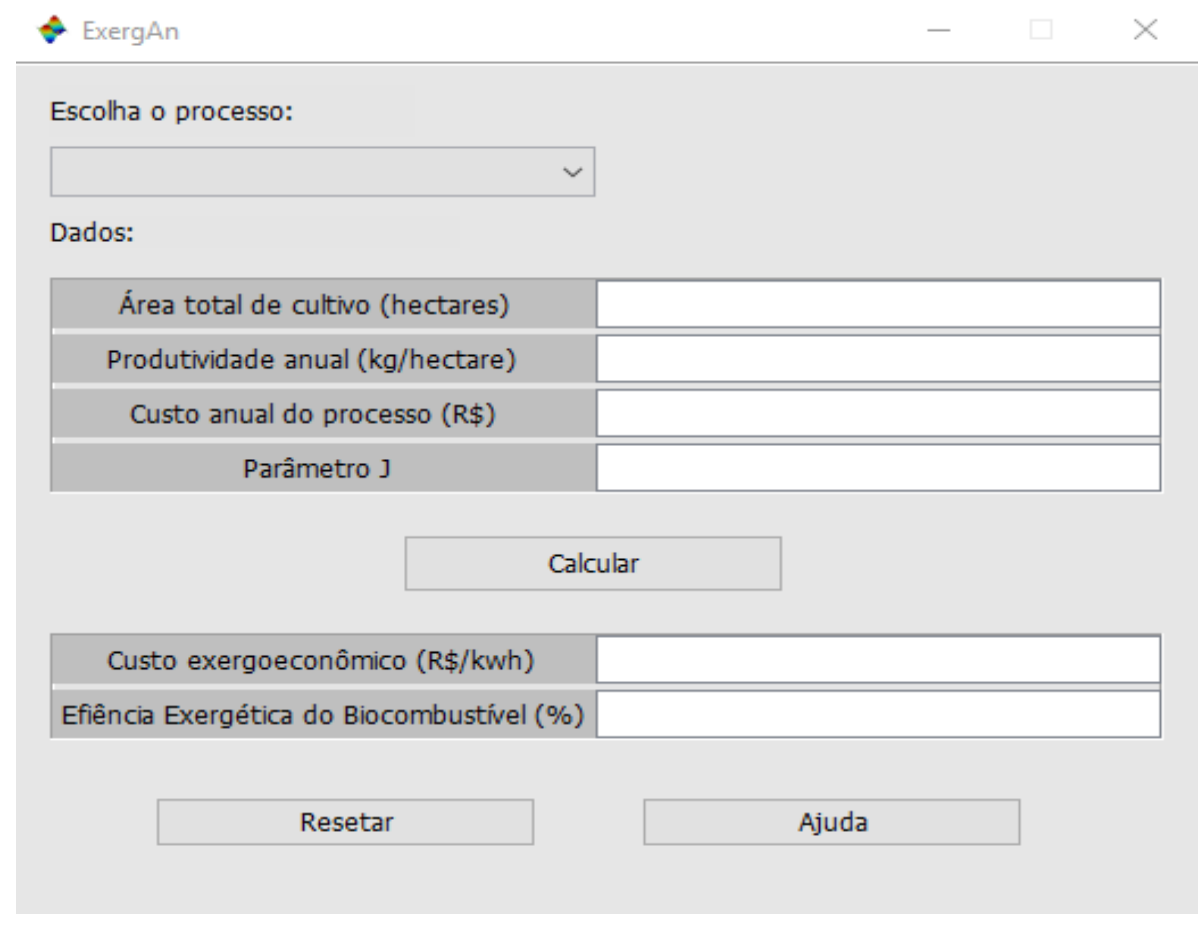

Figura 1: Interface gráfica do software ExergAn.

A aplicabilidade do programa ainda é bem restrita se comparada à grande variedade de cultura e de processos de produção de biocombustíveis que utilizam essas diferentes culturas. Nesse sentido, propõem-se algumas possibilidades de implementação do 
software, como adicionar novos processos de relevância no mercado, implementar novas variáveis de entrada, ou mesmo estabelecer novos parâmetros de saída capazes de comparar processos distintos. Como exemplo de funcionalidade, supondo um caso de produção de bioetanol, utilizando 1000 hectares de área total de cultivo, com uma produtividade anual média de $3300 \mathrm{~kg}$ por hectare, um custo anual total de 5 milhões de reais e com parâmetro $\mathrm{J}$ de $20 \%(0,2)$, espera-se um custo exergoeconômico aproximado de $9,60 R \$ / k w h$ e uma eficiência exergética de $8,22 \%$, ambas obtidas quando essa situação é simulada no processo, conforme mostra a figura a seguir:

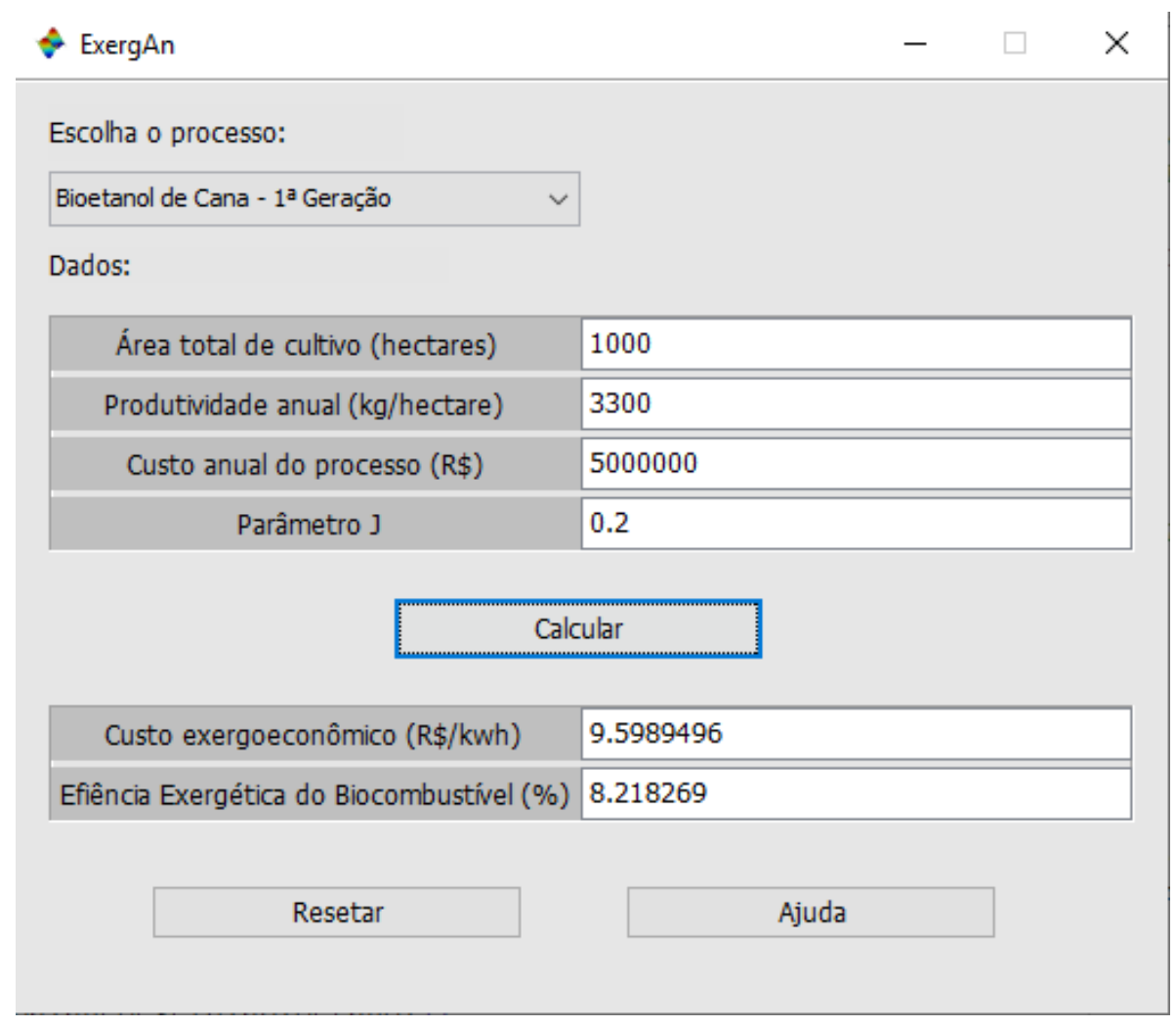

Figura 2: Cálculo da situação hipotética proposta.

A partir dessa situação, pode-se manter a área total de cultivo e modificar as outras variáveis para obter outras situações hipotéticas comparáveis ao caso exemplificado.

\section{$5 \quad$ CONCLUSÕES}

O software desenvolvido consegue realizar os cálculos e prover uma análise preliminar sobre os custos do combustível e sobre a eficiência exergética de sua produção. Os cálculos possuem limitações, mas podem se aproximar de uma situação real a partir da utilização do parâmetro $\mathrm{J}$, desde que se conheça dados que possam ser traduzidos nesse parâmetro.

Dificilmente os resultados calculados serão completamente parelhos à realidade, devido às considerações assumidas e às complexidades que os processos podem assumir. Portanto, afirma-se que os resultados obteníveis pelo software constituem uma ferramenta que pode complementar outras informações sobre a escolha de uma cultura de plantio e/ou de rota industrial para produção de biocombustíveis, sendo fundamental ao usuário buscar informações adicionais caso necessário. Assume-se que o software ainda é um protótipo, uma vez que há várias possibilidades de ampliar sua aplicabilidade e sua análise. 


\section{REFERÊNCIAS}

Carvalho, J.. Energia e sociedade. Estudos Avançados. v.28, n.82, p. 25-39, 2014.

Arredondo, H.I.V.; Junior, S. O.; Avaliação exergética e exergoambiental da produção de biocombustíveis. Tese (Doutorado em Engenharia Mecânica) - Escola Politécnica da Universidade de São Paulo, São Paulo, SP, 235p, 2009.

Azevedo, A. N. G; Lima, B. G. A. . Biocombustíveis: Desenvolvimento e Inserção Internacional. Revista Direito Ambiental e Sociedade. v. V6, p.77-100, 2016.

Kotas, T. J.; The exergy method of thermal plant analysis. Department of Mechanical Engineering, Queen Mary and Westfield College, University of London. Krieger Publishing Company, Malabar - Florida, 1995.

J. Szargut; Chemical Exergies of the Elements; Technical University of Silesia, Politechnika Ślaska. Instytut Techniki Cieplnej. Konarskiego 22. 44-100. Gliwice. Poland, 1989.

Cerqueira, S. A. A. G. Metodologias de Análise Termoeconômica de Sistemas. Tese (Doutorado em Engenharia Mecânica) - Faculdade de Engenharia Mecânica, Universidade Estadual de Campinas, Campinas, SP, 1999.

J. Szargut, D.R. Morris, F.R. Steward, Exergy analysis of thermal, chemical and metallurgical process. Hemisphere, New York, 1988.

Gomes, S. I. . Biomassa florestal para geração de energia em sistema de produção silvipastoril. Tese (Doutorado em Engenharia Química) - Universidade Estadual de Maringá, Departamento de Engenharia Química,Maringá, PR, 2017.

Lima, U. A. et al. Biotecnologia industrial: Processos fermentativos e enzimáticos. São Paulo: Blucher, v. 3, 2001.

Junior, S. O. . Exergy: Production, Cost and Renewability. 1. ed. [SI]: Springer-Verlag London, 2013.

Medeiros, J. F. . Análise de produção e purificação de biodiesel etílico de óleos de soja e canola por centrifugação. Dissertação (Mestrado em Engenharia Química) - 
Universidade Estadual de Maringá, Departamento de Engenharia Química, Maringá, PR, 2014.

Brandão, K. S. R. et al. Produção de Biodiesel por Transesterificação do Óleo de Soja com Misturas de Metanol-Etanol. Anais do I Congresso da Rede Brasileira de Tecnologia do Biodiesel, Brasília: Rede Brasileira de Tecnologia do Biodiesel, p. 141-146, 2007.

Mourad, A. L. ; Walter, A. The energy balance of soybean biodiesel in Brazil: a case study. Biofuels, Bioproducts and Biorefining, 5(2), p. 185-197, 2011.

Menezes, R. C. S. . Termodinâmica e o uso eficiente de recursos análise exergética de uma biorrefinaria de cana-de-açúcar. Tese (Doutorado em Ciências Mecânicas) Universidade de Brasília, Brasília, DF, 105 p, 2015.

Ojeda, K.; SANCHEZ, E.; KAFAROV, V. Sustainable ethanol production from lignocellulosic biomass - Application of exergy analysis. Energy, v. 36, p. 2119-2128, 2011b.

Almeida, E. L. ; ANDRADE, C. M. G; SANTOS, O. A. . Biodiesel production versus bioethanol production process. In: 10th International Conference on Sustainable Energy \& Environmental Protection, 2017, Bled. Prociedings of 10th International Conference on Sustainable Energy \& Environmental Protection: Bioenergy and Biofuels. Maribor: University of Maribor Press, p. 1-10, 2017.

Mari, J. et al. . Comparative Exergetic Analysis of Two Biodiesel Production Routes. DEStech Transactions on Engineering and Technology Research, v. 1, p. 402-406, 2018.

Almeida, E. L. ; ANDRADE, C. M. G. ; SANTOS, O. A. . Production of Biodiesel Via Catalytic Processes: A Brief Review. INTERNATIONAL JOURNAL OF CHEMICAL REACTOR ENGINEERING, v. 1, p. 1-13, 2018.

Mari, M. et al. Exergetic Analysis between a First-Generation Bioethanol Production Plant and a Second-Generation Plant Coupled to Conventional Process. DEStech Transactions on Engineering and Technology Research, v. 1, p. 407-412, 2018. 